\title{
Lie point symmetries and ODEs passing the Painlevé test
}

D. Levi, D. Sekera, P. Winternitz

To cite this article: D. Levi, D. Sekera, P. Winternitz (2018) Lie point symmetries and ODEs passing the Painlevé test, Journal of Nonlinear Mathematical Physics 25:4, 604617, DOI: https://doi.org/10.1080/14029251.2018.1503435

To link to this article: https://doi.org/10.1080/14029251.2018.1503435

Published online: 04 January 2021 


\title{
Lie point symmetries and ODEs passing the Painlevé test
}

\author{
D. Levi \\ Dipartimento di Matematica e Fisica, Roma Tre University and INFN, \\ Sezione Roma Tre, Via della Vasca Navale 84, 00146 Roma, Italy \\ levi@roma3.infn.it \\ D. Sekera \\ Institute of Physics, Laboratory for Particle Physics and Cosmology, \\ École Polytechnique Fédérale de Lausanne, CH-1015 Lausanne, Switzerland \\ david.sekera@epfl.ch \\ P. Winternitz \\ Département de mathématiques et de statistique and Centre de recherches mathématiques, \\ Université de Montréal, C.P. 6128, succ. Centre-ville, Montréal (QC) H3C 3J7, Canada \\ wintern@crm.umontreal.ca
}

Received 29 April 2018

Accepted 10 May 2018

\begin{abstract}
The Lie point symmetries of ordinary differential equations (ODEs) that are candidates for having the Painlevé property are explored for ODEs of order $n=2, \ldots, 5$. Among the 6 ODEs identifying the Painlevé transcendents only $P_{I I I}, P_{V}$ and $P_{V I}$ have nontrivial symmetry algebras and that only for very special values of the parameters. In those cases the transcendents can be expressed in terms of simpler functions, i.e. elementary functions, solutions of linear equations, elliptic functions or Painlevé transcendents occurring at lower order. For higher order or higher degree ODEs that pass the Painlevé test only very partial classifications have been published. We consider many examples that exist in the literature and show how their symmetry groups help to identify those that may define genuinely new transcendents.
\end{abstract}

\section{Introduction}

Two systematic methods exist for solving nonlinear ordinary differential equations (ODEs) or systems of ODEs. One is symmetry analysis, based on Lie group theory. The other is based on the study of the singularity structure of the solutions of the considered ODEs, namely Painlevé analysis.

The purpose of this article is to study the interplay between the two methods when both are applicable. Thus we shall consider one nonlinear ODE of the form

$$
y^{(n)}=R\left(x, y, y^{\prime}, \ldots, y^{(n-1)}\right)
$$

where $R$ is rational in $y, y^{\prime}, \ldots, y^{(n-1)}$ with coefficients that are analytic in $x$. We shall assume that (1.1) passes the Painlevé test in its original form [1-3,6,16,18,35,37,48], that is that its general solution can be written in the form

$$
y(x)=\sum_{n=0}^{\infty} a_{n}\left(x-x_{0}\right)^{n+\alpha}, \quad a_{o} \neq 0, \quad x_{0} \in \mathbb{C},
$$


were $\alpha$ is an integer (usually a negative one) and $x_{0}$ is an arbitrary point in the complex plane. The constants $a_{n}$ are to be determined from a recursion relation of the form

$$
P(k) a_{k}=\phi\left(a_{k-1}, \ldots, a_{0}, x_{0}\right),
$$

where $P(k)$ is a polynomial in $k$ that has $n-1$ non-negative integer roots, called resonances. The values of $a_{k}$ for $k$ at a resonance is arbitrary, but a resonance condition

$$
\phi\left(a_{k}, a_{k-1}, \ldots, a_{0}, x_{0}, \alpha\right)=0
$$

must be satisfied for each resonance value of $k$, identically in $x_{0}$ and in the values of $a_{\tilde{k}}$ where $\tilde{k}<k$ are all previous resonance values. If $n-1$ such resonances exist then the solution (1.2) depends on $n$ arbitrary constants $\left(x_{0}, a_{k}, a_{k_{1}}, a_{k_{2}}, \ldots, a_{k_{n-1}}\right)$ and may be the general solution. If (1.1) is of the polynomial type, i.e. $R$ is a polynomial in $y$ and its derivatives, the Painlevé test is complete. If $R=\frac{P}{Q}$ is the ratio of two polynomials $P$ and $Q$, then further expansions are necessary to investigate values of $x_{0}$ where the polynomial in the denominator, $Q\left(y, y^{\prime}, \ldots, y^{(n-1)}, x\right)$ vanishes.

Improvements of the Painlevé test have been proposed that provide further necessary conditions for an equation to have the Painlevé property $[17,29,30,38]$. In particular they analyse negative resonances and address the problem of essential singularities about which the solutions may be single valued (as opposed to the original Painlevé test which allows only poles). Moreover the term Painlevé property has been further refined [38].

The test provides necessary conditions for having the Painlevé property. To make it sufficient one would have to prove that the radius of convergence of the series (1.2) is positive and that arbitrary initial conditions can be satisfied.

Let us now assume that (1.1) passes the Painlevé test. The question is what further can be achieved by performing a symmetry analysis.

We will consider examples of ODEs of order up to 5 that pass the Painlevé test and show how a symmetry analysis can help to identify those for which we can reduce their order or find particular or general solutions. In cases when the ODEs involve parameters, symmetry analysis can identify parameter values for which the order of the ODEs can be reduced. The same comments apply to equations that have passed the modified Painlevé test [17,29,30,38] and are candidates for having the Painlevé property.

Part of our motivation is that in the study of superintegrable systems, i.e. systems with more integrals of motion than degrees of freedom exotic potentials keep occurring [4, 28, 33, 34, 42-44]. These are potentials that satisfy nonlinear ODEs of order $N$, where $N$ depends on the order of the integrals of motion as quantum mechanical operators. So far for integrals of order 3, 4, 5 it turns out that all exotic potentials that allow the representation of variables in $E_{2}$ in Cartesian or polar coordinates satisfy ODEs that pass the Painlevé test.

In Section 2 we shall look at the symmetry properties of the 50 classes of second order first degree ODEs with the Painlevé property obtained by Painlevé and Gambier. Examples of higher order ODEs are treated in Section 3. Throughout the text the equations and their solutions can be real or complex. Correspondingly their symmetry algebras will be considered over the field $\mathbb{F}=\mathbb{R}$ or $\mathbb{F}=\mathbb{C}$. 


\section{Second order ODEs with the Painlevé property and their Lie point symmetries}

First of all some comments on Lie point symmetries of ODEs are in order. The symmetry algebra of a given ODE will be realized by vector fields of the form

$$
\hat{X}=\xi(x, y) \partial_{x}+\phi(x, y) \partial_{y}
$$

where $\xi(x, y)$ and $\phi(x, y)$ are smooth functions on some open set $\{x, y\} \in \mathbb{C}^{2}$. For a given ODE

$$
F\left(x, y, y^{\prime}, \ldots, y^{(n)}\right)=0
$$

one obtains the functions $\xi(x, y)$ and $\phi(x, y)$ by solving the determining equations obtained using a standard algorithm $[7,41,49]$, now implemented in all major symbolic manipulation programs. For $n \geq 2$ the determining equations are an overdetermined system of linear partial differential equations (PDEs).

For second order ODEs the following results (due to Sophus Lie) are useful for our purposes.

Theorem 2.1 (Lie). A second order $O D E$

$$
F\left(x, y, y^{\prime}, y^{\prime \prime}\right)=0, \quad \frac{\partial F}{\partial y^{\prime \prime}} \neq 0,
$$

can have a symmetry algebra $L$ of dimension $\operatorname{dim} L=N$, with $N=0,1,2,3$, or 8 . For $N=1$ equation (2.3) can be reduced to a 1st order ODE. For $N=2$ equation (2.3) can be solved by quadratures. For $N=3$ the quadratures can be calculated explicitly. For $N=8$ the algebra is locally isomorphic to $\operatorname{sl}(3)$ and (2.3) may be transformed into the equation $\ddot{u}(t)=0$ (by a point transformation $(x, y) \rightarrow$ $(t, u)$ ).

\section{Comments}

(1) For $N=1,2,3$ the obtained solutions may be implicit.

(2) Every second order ODE linearizable by a point transformation has $\operatorname{dim} L=8$

For $n \geq 3$ we have the following results:

(1) The maximal dimension of the symmetry algebra of (2.2) for $n \geq 3$ is $N=n+4$. This is attained for the equation

$$
y^{(n)}=0
$$

and the corresponding vector fields are

$$
\left\{\partial_{x}, x \partial_{x}, x^{2} \partial_{x}+(n-1) x y \partial_{y}, y \partial_{y}, \text { and } x^{k} \partial_{y}, k=0, \ldots, n-1\right\} .
$$

It is however not true that any linear ODE of order $n$ has $\operatorname{dim} L=n+4$ and only a subclass of linear ODEs can be transformed into (2.4), namely precisely those with $\operatorname{dim} L=N+4$. Other possible dimensions for symmetry algebras of $n^{\text {th }}$ order linear ODEs are $n+1$ and $n+2$.

(2) If we have $\operatorname{dim} L=1$ or 2 , the order of (2.2) can be reduced by 1 or 2 , respectively. If we have $\operatorname{dim} L=N \geq 3$, the order of (2.2) can be reduced by $s \leq N$ where $s$ is the dimension of a maximal solvable subalgebra of $L$. 
Painlevé [50] and Gambier [31] classified all equations of the form (1.1) for $n=2$ that have the Painlevé property (no movable singularities other than poles) into 50 conjugacy classes under the (local) Lie group of (local) point transformations of $(x, y)$ that preserve the Painlevé property. These are arbitrary locally invertible transformations of $x$ and fractional linear transformations of $y$ $(x, y) \rightarrow(\tilde{x}, \tilde{y})$ with

$$
\tilde{x}=\phi(x), \quad \tilde{y}=\frac{\alpha(x) y+\beta(x)}{\gamma(x) y+\delta(x)}, \quad \alpha \delta-\beta \gamma \neq 0,
$$

where $\phi, \alpha, \beta, \gamma, \delta$ are arbitrary functions, smooth in some interval $x \in I$, such that the inverse transformation exists.

Among these 50 classes, listed in many books (see e.g. [27,36]), 6 define the famous Painlevé trascendents. All 50 equations, in their standard form, can be written as

$$
y^{\prime \prime}=\mathscr{L}(x, y)\left(y^{\prime}\right)^{2}+\mathscr{M}(x, y) y^{\prime}+\mathscr{N}(x, y),
$$

where $\mathscr{L}$ is rational in $y$ and has between 1 and 4 poles in $y$. One pole at $y=y_{0}$ is shifted to $y=\infty$. Then $\mathscr{L}$ takes one of the forms

$$
\mathscr{L}=\left\{0, \frac{a}{y}, \frac{b}{y}+\frac{c}{y-1}, \frac{1}{2}\left[\frac{1}{y}+\frac{1}{y-1}+\frac{1}{y-\lambda}\right]\right\}
$$

where $\lambda$ is arbitrary, but the residues $a$ and $b$ have specific values (like $a=1$, or $a=\frac{m-1}{m}$ with $m \geq 2$ and integer). The list of 50 equations is standardly ordered into 8 classes by increasing order of the number of poles in $\mathscr{L}$ as in (2.7).

We have calculated the Lie point symmetries for all 50 classes in the list and for our purposes a different order emerges. We follow the standard list given e.g. in Ince [36] and our notation for the classes of equations in Ince's list is $I_{1}, I_{2}, \ldots, I_{50}$.

(1) Autonomous equations. For these the dimension $N$ of the symmetry algebra $L$ satisfies $N=$ $1,2,3$ or 8 . Indeed the Lie algebra always contains the vector field $\hat{X}_{1}=\partial_{x}(\operatorname{dim} L=1)$. If $\operatorname{dim} L=8$ the algebra is isomorphic to $\operatorname{sl}(3, \mathbb{C})$ and the equation can be transformed into $\ddot{u}=0$. The ODEs in this class and the values of $N=\operatorname{dim} L$ are: $I_{1},(N=8) ; I_{2},(N=2) ; I_{3},(N=1)$; $I_{7},(N=2) ; I_{8},(N=1) ; I_{11},(N=8) ; I_{12},(N=1) ; I_{17},(N=8) ; I_{18},(N=2) ; I_{19},(N=1)$; $I_{21},(N=2) ; I_{22},(N=3) ; I_{23},(N=1) ; I_{29},(N=2) ; I_{30},(N=1) ; I_{32},(N=3) ; I_{33},(N=1)$; $I_{37},(N=8) ; I_{38},(N=1) ; I_{41},(N=8) ; I_{43},(N=8) ; I_{44},(N=1) ; I_{49},(N=1)$.

(2) Equation with explicit (polynomial) dependence on the independent variable $x . I_{4} \sim P_{I}$, $I_{9} \sim P_{I I}, I_{13} \sim P_{I I I}, I_{20}$ equivalent to special case of $P_{I I}, I_{31} \sim P_{I V}, I_{34}$ reducible to $P_{I I}, I_{39} \sim P_{V}$, $I_{50} \sim P_{V I}$. We see that all the irreducible Painlevé transcendents appear in this class. All of them, except $P_{I}$, depend on at least one parameter. For generic values of the parameters we have $N=0$. For specific values of the parameters there are some exceptions (see below).

(3) Equations involving arbitrary functions of $x$. These are equations, $I_{5}, I_{6}, I_{15}, I_{16}$ and $I_{24}$ with one arbitrary function $q(x)$ and $I_{14}, I_{25}, I_{27}$ and $I_{40}$ with two arbitrary functions each. Here Lie group theory is of little help, but Painlevé and Gambier integrated these equations by finding a first integral or presenting a non-point transformation $y=g(x, u, \dot{u})$ that ultimately reduces the given equation to a linear equation, or one for elliptic functions.

(4) Equations involving functions that satisfy specific differential equations. These are the cases $I_{10}, I_{26}, I_{28}, I_{35}, I_{36}, I_{42}, I_{46}, I_{47}$ and $I_{48}$ in Ince [36]. We treat cases $I_{10}$ and $I_{26}$ in detail. 
The remaining cases are very similar.

$$
\mathbf{I}_{\mathbf{1 0}} \quad y^{\prime \prime}=-y y^{\prime}+y^{2}-12 q y+12 q^{\prime}
$$

(a) $q=0, N=2$

(b) $q=q_{0} \neq 0,\left(q_{0}=\right.$ const. $), N=1$

(c) $q^{\prime \prime}=6 q^{2}, q \neq 0, N=1$

(d) $q^{\prime \prime}=6 q^{2}+1, q \neq q_{0}, N=1$

(e) $q^{\prime \prime}=6 q^{2}+x, N=0$

In cases (a) and (b) $I_{10}$ has constant coefficients and can be integrated by symmetry. In cases (c) and (d) the function $q$ is an elliptic function and $I_{10}$ can be reduced to a first order ODE (and to elliptic functions). In case (e) the function $q$ is itself a $P_{I}$ transcendent and $I_{10}$ is solved in terms of $P_{I}$.

$$
\mathbf{I} \mathbf{2 6} \quad y^{\prime \prime}=\frac{3}{4 y}\left(y^{\prime}\right)^{2}+\frac{6 q^{\prime}}{y} y^{\prime}+3 y^{2}+12 q y-12 q^{\prime \prime}-\frac{36\left(q^{\prime}\right)^{2}}{y}
$$

(a) $q^{\prime \prime}=6 q^{2}, q \neq 0, N=1$

(b) $q^{\prime \prime}=6 q^{2}+1 / 2, N=1$

(c) $q^{\prime \prime}=6 q^{2}+x, N=0$

Here again, in cases (a) and (b) $q$ is an elliptic function and the order of the equation can be decreased by one. In case (c) $q$ is a $P_{I}$ transcendent and so is the solution of $I_{26}$.

The remaining cases are quite similar. The ODEs involve one or more functions of $x$ that are all ultimately expressed in terms of one function. This function either satisfies an equation for elliptic functions, or for one of the irreducible Painlevé transcendents, namely $P_{I}, P_{I I}$ or $P_{I V}$. In the first case a nontrivial symmetry algebra exists (with $N=1$ ) and the ODE can be integrated in terms of elliptic or elementary functions. If $q(x)$ is itself a Painlevé transcendent, then so is $y$ and we have $\operatorname{dim} L=0$.

Let us now look at the 6 irreducible Painlevé transcendents in detail and calculate their Lie point symmetry algebras. We already know that for generic values of their parameters their Lie point symmetry algebras are $L=\{0\}$ in all cases. Applying the standard symmetry algebra algorithm we find that for $P_{I}, P_{I I}$ and $P_{I V}$ we have $L=\{0\}$ for all values of the parameters in $P_{I I}$ and $P_{I V}\left(P_{I}\right.$ has no parameters). For $P_{I I I}, P_{V}$ and $P_{V I}$ the situation is different. Let us consider the three cases separately.

(1) $\boldsymbol{P}_{\boldsymbol{I I I}}$. The ODE in its standard form is:

$$
y^{\prime \prime}=\frac{1}{y}\left(y^{\prime}\right)^{2}-\frac{1}{x} y^{\prime}+\frac{1}{x}\left(\alpha y^{2}+\beta\right)+\gamma y^{3}+\frac{\delta}{y}
$$

Nontrivial Lie algebras are obtained in the following cases

(a) $\alpha=\beta=\gamma=\delta=0$. We obtain $\operatorname{dim} L=8$ realized by the vector fields

$$
\begin{array}{r}
\hat{Y}_{1}=y \partial_{y}, \quad \hat{Y}_{3}=y \ln y \partial_{y}, \quad \hat{Y}_{5}=y \ln x \partial_{y}, \quad \hat{Y}_{7}=x \ln x \ln y \partial_{x}+y \ln ^{2} y \partial_{y}, \\
\hat{Y}_{2}=x \partial_{y}, \quad \hat{Y}_{4}=x \ln x \partial_{x}, \quad \hat{Y}_{6}=x \ln y \partial_{x}, \quad \hat{Y}_{8}=y \ln x \ln y \partial_{y}+x \ln ^{2} x \partial_{x}
\end{array}
$$

By a change of variables

$$
(x, y) \rightarrow(t, z), t=\ln x, z=\ln y, x=e^{t}, y=e^{z},
$$


we transform the algebra $s l(3)$ into a more familiar form, namely

$$
\begin{aligned}
& \hat{Y}_{1}=\partial_{z}, \quad \hat{Y}_{3}=z \partial_{z}, \quad \hat{Y}_{5}=t \partial_{z}, \quad \hat{Y}_{7}=z\left(t \partial_{t}+z \partial_{z}\right), \\
& \hat{Y}_{2}=\partial_{t}, \quad \hat{Y}_{4}=t \partial_{t}, \quad \hat{Y}_{6}=z \partial_{t}, \quad \hat{Y}_{8}=t\left(t \partial_{t}+z \partial_{z}\right) .
\end{aligned}
$$

This is the Lie algebra of the group of projective transformations of the Euclidean plane $E_{2}$ (over $\mathbb{C}$ or $\mathbb{R}$ ). Eq. (2.10) is transformed into

$$
z_{t t}=e^{t}\left(\alpha e^{z}+\beta e^{-z}\right)+e^{2 t}\left(\gamma e^{2 z}+\delta e^{-2 z}\right),
$$

so for $\alpha=\beta=\gamma=\delta=0$ we obtain

$$
z_{t t}=0, \quad z(t)=A t+B,
$$

with symmetry algebra $s l(3)$ realized as (2.13). Transforming the solution (2.15) back to the original variables, we obtain

$$
y=c_{1} x^{c_{2}}, \quad\left(c_{1}=e^{B}, c_{2}=A\right) .
$$

The other cases with a nontrivial symmetry algebra are

(b) $\alpha \neq 0, \beta=\gamma=\delta=0, \operatorname{dim} L=2$

$$
\hat{A}=\hat{Y}_{1}-\hat{Y}_{2}=\partial_{z}-\partial_{t}, \quad \hat{B}=\hat{Y}_{4}-\hat{Y}_{5}-2 \hat{Y}_{1}=-t\left(\partial_{z}-\partial_{t}\right)-2 \partial_{z}
$$

(c) $\beta \neq 0, \alpha=\gamma=\delta=0, \operatorname{dim} L=2$

$$
\hat{A}=\hat{Y}_{1}+\hat{Y}_{2}=\partial_{z}+\partial_{t}, \quad \hat{B}=\hat{Y}_{4}+\hat{Y}_{5}+2 \hat{Y}_{1}=t\left(\partial_{z}+\partial_{t}\right)+2 \partial_{z}
$$

(d) $\gamma \neq 0, \beta=\alpha=\delta=0, \operatorname{dim} L=2$

$$
\hat{A}=\hat{Y}_{1}-\hat{Y}_{2}=\partial_{z}-\partial_{t}, \quad \hat{B}=\hat{Y}_{4}-\hat{Y}_{5}-\hat{Y}_{1}=-t\left(\partial_{z}-\partial_{t}\right)-\partial_{z}
$$

(e) $\delta \neq 0, \beta=\gamma=\alpha=0, \operatorname{dim} L=2$

$$
\hat{A}=\hat{Y}_{1}+\hat{Y}_{2}=\partial_{z}+\partial_{t}, \quad \hat{B}=\hat{Y}_{4}+\hat{Y}_{5}+\hat{Y}_{1}=t\left(\partial_{z}+\partial_{t}\right)+\partial_{z}
$$

(f) $\beta=\delta=0, \alpha \neq 0, \gamma \neq 0, \operatorname{dim} L=1, \hat{A}=\hat{Y}_{1}-\hat{Y}_{2}=\partial_{z}-\partial_{t}$,

(g) $\alpha=\gamma=0, \beta \neq 0, \delta \neq 0, \operatorname{dim} L=1, \hat{A}=\hat{Y}_{1}+\hat{Y}_{2}=\partial_{z}+\partial_{t}$.

The two dimensional algebra in all the above cases satisfies $[\hat{A}, \hat{B}]=\hat{A}$.

To integrate the ODE corresponding to cases $(\mathrm{b}), \cdots,(\mathrm{g})$ above we need a further transformation $(t, z) \rightarrow(s, u)$ such that

$$
\hat{A}=\partial_{u}, \quad \hat{B}=s \partial_{s}+u \partial_{u},
$$

to take (2.14) with the parameters appropriately specified in each case to its standard form. This is $\ddot{u}=F(s, \dot{u})$ for $\operatorname{dim} L=1, \ddot{u}=\frac{1}{s} F(\dot{u})$ for $\operatorname{dim} L=2$, where for (2.10) $F$ is some specific function. Alternatively, once the values of $\alpha, \beta, \gamma, \delta$ have been specified, one can simply ask Maple to integrate (2.10). Indeed the Lie group procedure has been built into the routines for analytically solving ODEs. The Maple procedure is based on the articles [13, 14].

In all cases the solutions are elementary and quite explicit. For example, for case (b) the two dimensional Lie algebra leads to the solution

$$
y(x)=\frac{1}{2 c_{1}^{2} \alpha x}\left(\tan ^{2}\left(\frac{\ln (x)+c_{2}}{2 c_{1}}\right)+1\right)
$$


(2) $\boldsymbol{P}_{\boldsymbol{V}}$. The ODE is

$$
y^{\prime \prime}=\left(\frac{1}{2 y}+\frac{1}{y-1}\right)\left(y^{\prime}\right)^{2}-\frac{1}{x} y^{\prime}+\frac{(y-1)^{2}}{x^{2}}\left(\alpha y+\frac{\beta}{y}\right)+\gamma^{y}+\delta \frac{y(y+1)}{y-1}
$$

Nontrivial Lie point symmetry algebras are obtained in the following cases

(a) $\alpha=\beta=\gamma=\delta=0 ; \operatorname{dim} L=8$

The algebra is realized by the vector fields

$$
\begin{aligned}
& \hat{Y}_{1}=\sqrt{y}(y-1) \partial_{y}, \quad \hat{Y}_{3}=\sqrt{y}(y-1) \operatorname{arctanh}(\sqrt{y}) \partial_{y}, \\
& \hat{Y}_{2}=x \partial_{x}, \quad \hat{Y}_{4}=x \ln x \partial_{x}, \quad \hat{Y}_{5}=\ln x \sqrt{y}(y-1) \partial_{y}, \\
& \hat{Y}_{6}=x \operatorname{arctanh}(\sqrt{y}) \partial_{x}, \\
& \hat{Y}_{7}=-\frac{1}{2} x \ln x \operatorname{arctanh}(\sqrt{y}) \partial_{x}+\sqrt{y}(y-1) \operatorname{arctanh}^{2}(\sqrt{y}) \partial_{y}, \\
& \hat{Y}_{8}=-\frac{1}{2} x \ln ^{2} x \partial_{x}+(y-1) \sqrt{y} \ln x \operatorname{arctanh}(\sqrt{y}) \partial_{y} .
\end{aligned}
$$

This realization of $\operatorname{sl}(3)$ is taken into the standard form (2.13) by the transformation

$$
(x, y) \rightarrow(t, z), t=\ln x, z=2 \operatorname{arctanh}(\sqrt{y}), x=e^{t}, y=\tanh ^{2} \frac{z}{2} .
$$

The transformation (2.21) take (2.19) into $z_{t t}=0$ so we obtain the solution $z=A t+B$ which in this case means

$$
y(x)=\tanh ^{2}\left(\frac{A}{2} \ln x+B\right)=\left(\frac{x^{2 A} e^{2 B}-1}{x^{2 A} e^{2 B}+1}\right)^{2}
$$

(we see the movable double pole at $x=-e^{-\frac{B}{A}}$ ).

The other special case with a nontrivial symmetry algebra is

(b) $\gamma=\delta=0,(\alpha, \beta) \neq(0),), \operatorname{dim} L=1, \hat{Y}=x \partial_{x}$

This again leads to an elementary solution.

(3) $\boldsymbol{P}_{\boldsymbol{V} \boldsymbol{I}}$. The ODE is

$$
\begin{aligned}
\ddot{y} & =\frac{1}{2}\left(\frac{1}{y}+\frac{1}{y-1}+\frac{1}{y-x}\right) \dot{y}^{2}-\left(\frac{1}{x}+\frac{1}{x-1}+\frac{1}{y-x}\right) \dot{y} \\
& +\frac{y(y-1)(y-x)}{x^{2}(x-1)^{2}}\left[\alpha+\beta \frac{x}{y^{2}}+\gamma \frac{x-1}{(y-1)^{2}}+\delta \frac{x(x-1)}{(y-x)^{2}}\right]
\end{aligned}
$$

The determining equations for the symmetry algebra are quite complicated in this case. So we used the Rif (Reduced involutive form) simplification algorithm contained in the Maple symbolic manipulation program $[52,53]$ and we obtain that only for the values of the parameters

$$
\alpha=\beta=\gamma=0, \quad \delta=\frac{1}{2}
$$

the determining equations for the symmetries are solvable and tell us that the dimension of the symmetry algebra is $\operatorname{dim} L=8$.

The linearizing transformation is known $[15,31,45,50,51]$ and involves elliptic integrals and solutions of the Heun equation. 


\section{Higher order ODEs}

The theory and specially the classification of ODEs of order $n \geq 3$ with the Painleve property is much less developed than for $n=2$.

Early work is due to Bureau [8-10], mainly on the case $n=3$. Much of the subsequent work was concentrated on equations in the Bureau polynomial class where the function $R$ in (1.1) is assumed to be polynomial, rather than a more general rational function. Classical results are due to Chazy $[11,12]$ and Garnier [32]. More recent is a very useful series of articles by Cosgrove [19-26]. For ODEs that go beyond the polynomial class see e.g. Mugan et. al. [5,46,47] and Kudryashov [39,40].

Here we shall just concentrate on some examples that are candidates for being new higher order Painlevé transcendents. We do not consider those that have already been integrated, or that have obvious symmetries (like autonomous equations with their $\hat{X}=\partial_{x}$ translational symmetry). In all considered cases it turns out that the symmetry algebra contains only translations in $x$ and dilations in $x$ and $y$, i.e. elements of the form $\hat{X}=\partial_{x}$ and $\hat{Y}=A x \partial_{x}+B y \partial_{y}$ Where $A$ can be restricted to $A=1$ or $A=0$ and $B$ is a number to be specified in each case. The corresponding group transformations are

$$
\tilde{x}=e^{\lambda A} x, \quad \tilde{y}=e^{\lambda B} y
$$

where $\lambda$ is a group parameter.

Third order ODEs. All third order ODEs in the polynomial class that have the Painlevé property have already been integrated in terms of lower order transcendents (see [23] and reference therein). We shall concentrate on examples of nonpolynomial ones given by Mugan and Jrad in [47]. They considered ODEs of the form

$$
y^{\prime \prime \prime}=c_{1} \frac{y^{\prime} y^{\prime \prime}}{y}+c_{2} \frac{\left(y^{\prime}\right)^{3}}{y^{2}}+F\left(y, y^{\prime}, y^{\prime \prime} ; x\right)
$$

where $c_{1}$ and $c_{2}$ are constants with $\left(c_{1}, c_{2}\right) \neq(0,0)$. They found many such equations and integrated most of them. Others are autonomous, so their order can be decreased by at least one. Notice that for any values of the constants $c_{1}$ and $c_{2}$ the first three terms in (3.2) transform identically under dilations, i.e. they are multiplied by $e^{(A-B) \lambda}$. Hence for $\mathrm{F}=0$ the symmetry algebra is three-dimensional: $\left\{\hat{X}_{1}=\partial_{x}, \hat{X}_{2}=x \partial_{x}, \hat{X}_{3}=y \partial_{y}\right\}$.

Let us look at some of the remaining ones. The $k_{i}$ are constants.

(1)

$$
\begin{aligned}
y^{\prime \prime \prime}= & 4 \frac{y^{\prime} y^{\prime \prime}}{y}-3 \frac{\left(y^{\prime}\right)^{3}}{y^{2}}+y^{2} y^{\prime}+\frac{\left(k_{3} x+k_{2}\right) y^{\prime}}{y}+\frac{k_{1} y^{\prime}}{y^{2}} \\
& -\frac{3}{4} \frac{k_{3}\left(k_{3} x+k_{2}\right) y}{k_{1}}-k_{3}
\end{aligned}
$$

[Eq. (2.67) in [47]]

For $\left(k_{1}, k_{3}\right) \neq(0,0)$ no symmetry.

For $k_{1} \neq 0$ and $k_{3}=0 \hat{X}=\partial_{x}$.

For $k_{1} \neq 0$ and $k_{2}=k_{3}=0$ we have the symmetry algebra $\left\{\hat{X}_{1}=\partial_{x}, \hat{X}_{2}=y \partial_{y}-x \partial_{x}\right\}$. 
(2)

$$
y^{\prime \prime \prime}=\frac{y^{\prime} y^{\prime \prime}}{y}-2 y y^{\prime \prime}+y^{2} y^{\prime}+y^{4}+\left(k_{2} x+k_{3}\right) y^{2}+k_{2}\left(\frac{2 y^{\prime}}{y}+y\right)
$$

[Eq. (2.106) in [47]]

For general $k_{2}, k_{3}$ : no symmetry.

For $k_{2}=0, k_{3}$ arbitrary: $\hat{X}=\partial_{x}$.

For $k_{2}=k_{3}=0$ we have the symmetry algebra $\left\{\hat{X}_{1}=\partial_{x}, \hat{X}_{2}=x \partial_{x}-y \partial_{y}\right\}$.

(3)

$$
y^{\prime \prime \prime}=3 \frac{y^{\prime} y^{\prime \prime}}{y}-\frac{16\left(y^{\prime}\right)^{3}}{9 y^{2}}+\left(k_{1} x+k_{2}\right) y^{\prime}+k_{2} y=0
$$

[Eq. (4.6) in [47]]

For any $k_{1}$ and $k_{2}$ we have the symmetry $\hat{X}=y \partial_{y}$. The transformation $y=e^{u}$ takes $\hat{X}$ into $\hat{X}=\partial_{u}$. Putting $u_{x}=3 w$ we reduce (3.5) to the Painlevé transcendent $P_{I I}$ for $k_{1} \neq 0$. For $k_{1}=1$ the algebra is $\left\{\hat{X}_{1}=\partial_{x}, \hat{X}_{2}=y \partial_{y}\right\}$ and the ODE reduces to that for elliptic functions.

(4)

$$
y^{\prime \prime \prime}=\frac{y^{\prime} y^{\prime \prime}}{y}-24 y^{2}+k_{1} y+\left(\frac{k_{1}^{2}}{12} x+k_{2}\right) \frac{y^{\prime}}{y}-\frac{k_{1}^{2}}{12}
$$

[Eq. (4.14) in [47]]

For $k_{1}, k_{2}$ general, no symmetries.

For $k_{1}=0, \hat{X}_{1}=\partial_{x}$

For $k_{1}=k_{2}=0$ we have the symmetry algebra $\left\{\hat{X}_{1}=\partial_{x}, \hat{X}_{2}=x \partial_{x}-3 y \partial_{y}\right\}$.

We see that (3.3), (3.4) and (3.6) survive as candidates for new Painlevé transcendents (for generic values of the parameters $k_{i}$ ), whereas (3.5) is integrated in terms of lower order transcendents.

ODEs of order 4 not in polynomial class. Kudryashov does not perform a classification of higher order equations with the Painlevé property. Instead he present an ingeneous method for generating such equations. In [40] he present 4 such fourth order equations. One (his eq. (3.7)) is in the polynomial class. We shall concentrate on the remaining three.

(1)

$$
\begin{aligned}
& y^{\prime \prime \prime \prime}-3 \frac{y^{\prime} y^{\prime \prime}}{y}-\frac{7}{2} \frac{\left(y^{\prime \prime}\right)^{2}}{y}+\frac{17}{2} \frac{\left(y^{\prime}\right)^{2} y^{\prime \prime}}{y^{2}}-\frac{27}{8} \frac{\left(y^{\prime}\right)^{4}}{y^{3}}+\left(\beta-\frac{5 \delta}{y}\right) y^{\prime \prime} \\
& -\frac{1}{2}\left(\frac{\beta}{y}-\frac{15 \delta}{y^{3}}\right)\left(y^{\prime}\right)^{2}+2 \gamma y^{2}-2 \alpha x y+\frac{\beta \delta}{y}-\frac{3 \delta^{2}}{2 y^{3}}=0
\end{aligned}
$$

[Eq. 3.14 in [40]]

For $\alpha, \beta, \gamma, \delta$ generic there are no symmetries.

For $\alpha \neq 0, \beta$ arbitrary, $\gamma=\delta=0$ the algebra is $\hat{X}=y \partial_{y}$.

For $\alpha=0$. $\beta \neq 0, \gamma \neq 0, \delta=0$ we have the symmetry algebra $\left\{\hat{X}_{1}=\partial_{x}, \hat{X}_{2}=x \partial_{x}-4 y \partial_{y}\right\}$.

For $\alpha=\beta=\gamma=0, \delta \neq 0$ we have the symmetry algebra $\left\{\hat{X}_{1}=\partial_{x}, \hat{X}_{2}=x \partial_{x}+y \partial_{y}\right\}$.

For $\alpha=\beta=\gamma=\delta=0$ we have the symmetry algebra $\left\{\hat{X}_{1}=\partial_{x}, \hat{X}_{2}=x \partial_{x}, \hat{X}_{3}=y \partial_{y}\right\}$. 
(2)

$$
\begin{aligned}
& y^{\prime \prime \prime \prime}-4 \frac{y^{\prime} y^{\prime \prime \prime}}{y}-3 \frac{\left(y^{\prime \prime}\right)^{2}}{y}+\frac{21}{2} \frac{\left(y^{\prime}\right)^{2} y^{\prime \prime}}{y^{2}}-\frac{9}{2} \frac{\left(y^{\prime}\right)^{4}}{y^{3}}-\left(2 \alpha x+\frac{5 \delta}{y^{2}}\right) y^{\prime \prime} \\
& +2\left(\frac{\alpha x}{y}+\frac{5 \delta}{y^{3}}\right)\left(y^{\prime}\right)^{2}+\beta y^{2}-2 \alpha y^{\prime}+\gamma-4 \frac{\alpha \delta x}{y}-2 \frac{\delta^{2}}{y^{3}}=0
\end{aligned}
$$

[Eq. 3.28 in [40]]

For $\alpha, \beta, \gamma, \delta$ generic there are no symmetries.

For $\alpha \neq 0, \beta=\gamma=\delta=0$ the algebra is $\hat{X}=y \partial_{y}$.

For $\alpha=0 . \beta \neq 0, \gamma=\delta=0$ we have the symmetry algebra $\left\{\hat{X}_{1}=\partial_{x}, \hat{X}_{2}=x \partial_{x}-4 y \partial_{y}\right\}$.

For $\alpha=\beta=0, \gamma \neq 0, \delta=0$ we have the symmetry algebra $\left\{\hat{X}_{1}=\partial_{x}, \hat{X}_{2}=x \partial_{x}+y \partial_{y}\right\}$.

For $\alpha=\beta=\gamma=\delta=0$ we have the symmetry algebra $\left\{\hat{X}_{1}=\partial_{x}, \hat{X}_{2}=x \partial_{x}, \hat{X}_{3}=y \partial_{y}\right\}$.

(3)

$$
\begin{aligned}
& y^{\prime \prime \prime \prime}-2 \frac{y^{\prime} y^{\prime \prime \prime}}{y}-5 y^{2} y^{\prime \prime}-\frac{5}{2} y\left(y^{\prime}\right)^{2}-\frac{3}{2} \frac{\left(y^{\prime \prime}\right)^{2}}{y}+2 \frac{\left(y^{\prime}\right)^{2} y^{\prime \prime}}{y^{2}}-2 \frac{\alpha x y^{\prime \prime}}{y} \\
& +2 \frac{\alpha x\left(y^{\prime}\right)^{2}}{y^{2}}-2 \frac{\alpha y^{\prime}}{y}+\frac{5}{2} y^{5}-\beta^{2} y^{3}+2 \alpha x y^{2}+\gamma y-\frac{1}{2} \frac{\alpha^{2} x^{2}}{y}=0
\end{aligned}
$$

[Eq. 4.6 in [40]]

For $\alpha \neq 0$ no symmetry.

For $\alpha=0 \hat{X}=\partial_{x}$.

For $\alpha=0, \beta$ and $\gamma$ arbitrary, the algebra is $\hat{X}=\partial_{x}$.

For $\alpha=\gamma=0, \beta$ arbitrary we have the symmetry algebra $\left\{\hat{X}_{1}=\partial_{x}, \hat{X}_{2}=x \partial_{x}-y \partial_{y}\right\}$.

ODEs of order 4 and 5 in the polynomial class. We use the Cosgrove classification presented in [24] and [25] and calculate the Lie point symmetries of the ODEs that he identifies as defining new transcendents. Specifically these are equations

$$
\begin{gathered}
\boldsymbol{F}_{\boldsymbol{V}}: \quad y^{\prime \prime \prime \prime}=20 y y^{\prime \prime}+10\left(y^{\prime}\right)^{2}-40 y^{3}+\alpha y+k x+\beta \\
\boldsymbol{F}_{\boldsymbol{V} \boldsymbol{I}}: \quad y^{\prime \prime \prime \prime}=18 y y^{\prime \prime}+9\left(y^{\prime}\right)^{2}-24 y^{3}+\alpha y^{2}+\frac{1}{9} \alpha^{2} y+k x+\beta \\
\boldsymbol{F}_{\boldsymbol{X V I I}}: \quad y^{\prime \prime \prime \prime}=10 y^{2} y^{\prime \prime}+10 y\left(y^{\prime}\right)^{2}-6 y^{5}-10 \delta\left(y^{\prime \prime}-2 y^{3}\right)+(\lambda x+\mu) y+\sigma \\
\boldsymbol{F}_{\boldsymbol{X V I I I}}: \quad y^{\prime \prime \prime \prime}=-5 y^{\prime} y^{\prime \prime}+5 y^{2} y^{\prime \prime}+5 y\left(y^{\prime}\right)^{2}-y^{5}+(\lambda x+\alpha) y+\gamma \\
\boldsymbol{F i f}_{\boldsymbol{I}}: \quad y^{\prime \prime \prime \prime \prime \prime}=18 y y^{\prime \prime \prime}+36 y^{\prime} y^{\prime \prime}-72 y^{2} y^{\prime}+3 \lambda y^{\prime \prime}+\frac{1}{2} \lambda x\left(5 y^{\prime \prime \prime}-36 y y^{\prime}\right) \\
+\frac{1}{x}\left\{y^{\prime \prime \prime \prime \prime}-18 y y^{\prime \prime}-9\left(y^{\prime}\right)^{2}+24 y^{3}-3 \lambda y^{\prime}+k\right\}-\frac{1}{2} \lambda^{2} x\left(2 x y^{\prime}+y\right)
\end{gathered}
$$

All of them depend on 2 or 3 parameters and for general values of the parameters none of them has a nontrivial symmetry algebra.

For specific values of the parameters, the situation is as follows:

Eq. (3.10): $\alpha=\beta=k=0, \quad \hat{A}=\partial_{x}, \hat{B}=x \partial_{x}-2 y \partial_{y}$ 
$k=0, \alpha, \beta$ arbitrary, $\hat{A}=\partial_{x}$

Eq. (3.11): $\alpha=\beta=k=0, \quad \hat{A}=\partial_{x}, \hat{B}=x \partial_{x}-2 y \partial_{y}$ $k=0, \alpha, \beta$ arbitrary, $\hat{A}=\partial_{x}$

Eq. (3.12): $\lambda=\mu=\delta=\sigma=0, \quad \hat{A}=\partial_{x}, \hat{B}=x \partial_{x}-y \partial_{y}$ $\lambda=0, \delta, \mu, \sigma$ arbitrary, $\hat{A}=\partial_{x}$

Eq. (3.13): $\alpha=\lambda=\gamma=0, \quad \hat{A}=\partial_{x}, \hat{B}=x \partial_{x}-y \partial_{y}$

$\lambda=0, \alpha, \gamma$ arbitrary, $\hat{A}=\partial_{x}$

Eq. (3.14): $\lambda=k=0, \quad \hat{A}=x \partial_{x}-2 y \partial_{y}$

The situation is very similar to that of $P_{I I I}$ and $P_{V}$ for second order ODEs. In general these ODEs define new transcendents. For special values of the parameters Lie group theory makes it possible to reduce to lower order.

\section{Conclusions}

From the analysis of ODEs passing the Painlevé test we can draw the following conclusions valid for ODEs of any order.

(1) If an ODE that is a candidate for having the Painlevé property has a symmetry algebra of dimension $\operatorname{dim} L \geq 1$ it can by a change of variables be reduced to an ODE of lower order. The reduction $(x, y) \rightarrow(t, u)$ does not necessarily preserve the Painlevé property. However, if the reduced equation can be solved for $u(t)$, then the inverse transformation $u(t) \rightarrow y(x)$ will produce solutions of the original equations and the Painlevé property is restored. In any case, if we are looking for new Painlevé transcendents, not expressible in terms of lower order ones, only equations with $L=\{0\}$ need to be considered.

(2) Autonomous equations $y^{(n)}(x)=R\left(y, y^{\prime}, \ldots, y^{(n-1)}\right)$ always have at least a one dimensional symmetry algebra, $L=\left\{\partial_{x}\right\}$. Hence autonomous equations can never produce new transcendents.

(3) If a non-autonomous equation depends on parameters and in general has $L=\{0\}$, then group analysis can pick values of the parameters for which we have $\operatorname{dim} L \geq 1$. For those values the order of the ODE can be reduced and sometimes solutions can be obtained in terms of known functions.

(4) To our knowledge the Lie point symmetries of the 6 Painlevé transcendents for special values of the parameters have not been studied, or at least published, before. The result is not particularly important, since the obtained classical solutions are among those obtained by other more powerful methods (see e.g. [35, 54]).

However for higher order Painlevé type equations no such analysis is available and Lie point symmetries will provide a very useful starting point. Indeed in any singularity classification of ODEs it would be useful to add a classification of their symmetry algebras in the generic case and to identify special cases when the symmetry algebra is larger.

A complete classification of ODEs of the form (1) that have the Painleve property exists only for $n=1$, and $n=2$. For $n>2$ the classifications are very incomplete and most authors concentrated on the polynomial class of equations [8-10,19-26]. That notwithstanding, higher order ODEs passing the Painlevé test keep appearing in physical applications, in particular as superintegrable potentials in quantum mechanics, as mentioned in the Introduction. In quantum mechanics it is usually assumed that the integrals are polynomials of order $N$ in the momenta, i.e. differential operators of order $N$. For $N \geq 3$ exotic potentials start appearing. They do not satisfy any linear differential 
equation, only nonlinear ones. For $N=3,4,5$ it turned out that these equations always passed the Painlevé test. For $N=3$ and $N=4$ the equations were integrated in terms of known (second order) Painlevé transcendents. For $N \geq 5$ new irreducible higher order transcendents start appearing. It has been conjectured that superintegrable exotic potentials with integrals of any order will have the Painlevé property $[4,28,43]$.

It is doubtful that the complete classification of Painlevé type equations can be pushed much further. This makes it all the more important to have tools for obtaining special solution and for lowering the order of the nonlinear equations.

In this article we have restricted ourselves to Lie point symmetries. We have shown that they can help to identify Painlevé type equations that lead to lower order transcendents, to identify cases when the nonlinear equation can be linearized and to find particular "invariant" solutions.

More general symmetry transformations, for instance Lie-Bäcklund ones, should give further results. In particular they give many families of classical solutions of the original Painlevé equations $P_{I I}, \ldots, P_{V I}$ (for special values of the parameters) [35].

After completing this manuscript (and publishing its preprint as arXiv: 1712.09811v1) we became aware of a preprint by Contatto and Dunajski [15]. They address and solve a different problem, namely which of the second order ODEs having the Painlevé property are metrisable (i.e. their integral curves are geodesics of a pseudo Riemannian metric on some surface). In the Appendix we present the relation between their and our results.

Acknowledgments. DL has been supported by INFN IS-CSN4 Mathematical Methods of Nonlinear Physics. The research of PW was partially supported by a discovery research grant from NSERC of Canada. DL thanks the CRM for the support during his stay in Montréal where this research has been carried out. We thank M. Dunajski for calling the preprint [15] to our attention and for very helpful (electronic) discussions. We thank E. Cheb-Terrab for helping us in running the Rif program.

\section{Appendix}

In this Appendix we present the interesting relation between the results, contained in [15], from now on indicated as their results and our results.

Their results are summed up in Theorem 1.3 and in five points (bullets) in the conclusions of [15]. Let us compare them with the results presented here.

The Theorem 3.1 of [15] concerns the 6 irreducible Painleve transcendents and states that the only metrisable ones are $P_{I I I}$ for $\alpha=\gamma=0$, or $\beta=\delta=0, P_{V}$ for $\gamma=\delta=0$ and $P_{V I}$ for $\alpha=\beta=\gamma=0$ and $\delta=\frac{1}{2}$. Moreover the projective structure is flat for $P_{I I I}$ and $P_{V}$ with $\alpha=\beta=\gamma=\delta=0$ and for $P_{V I}$ with $\alpha=\beta=\gamma=0, \delta=\frac{1}{2}$.

Our result is $\operatorname{dim} L=8$ for $P_{V I}$ iff $\alpha=\beta=\gamma=0, \delta=\frac{1}{2}$ and for $P_{I I I}$ and $P_{V}$ with $\alpha=\beta=\gamma=$ $\delta=0$. We obtain $\operatorname{dim} L=1$ or 2 in the other metrisable cases.

The results contained in [15] for all 50 equations are related to ours as follows (we follow the list contained in [15] in Section 4).

(1) Metrisable with $4>m>1$. In our list they all have $\operatorname{dim} L=1$ or 2 and are in our Class 1 (autonomous equations).

(2) Metrisable with $m=4$. In our list in Class 1 but with $\operatorname{dim} L=3$. 
(3) Not metrisable, with a degenerate solution. We have $\operatorname{dim} L=0$ in all cases. We mention that $I_{14}$ is reducible to a Riccati equation and is in our Class 2. The other cases, $I_{20}$ and $I_{34}$ are in our Class 3 and are equivalent to the transcendent $P_{I I}$.

(4) Not metrisable. Class 3 and 4 in our list. They all involve unknown functions. In general have $\operatorname{dim} L=0$ but may be nontrivial for some specific functions.

(5) Metrisable and projectively flat. In our list: $\operatorname{dim} L=8(L \sim s l(3, \mathbb{R}))$. All in our Class 1 except $I_{6}$ which is in Class 3 .

\section{References}

[1] M.J. Ablowitz, A. Ramani and H. Segur, Nonlinear evolution equations and ordinary differential equations of Painlevé type, Lett Nuovo Cimento 23 (1978) 333-338.

[2] M.J. Ablowitz, A. Ramani and H. Segur, A connection between nonlinear evolution equations and ordinary differential equations of P-type. I, J. Math. Phys. 21 (1980) 715-721.

[3] M.J. Ablowitz, A. Ramani and H. Segur, A connection between nonlinear evolution equations and ordinary differential equations of P-type. II, J. Math. Phys. 21 (1980) 1006-1015.

[4] I. Abouamal and P. Winternitz, Fifth-order superintegrable quantum system separating in Cartesian coordinates. Doubly exotic potentials, J. Math. Phys. 59 (2018) 022104.

[5] Y. Adjabi, F. Jrad, A. Kessi, U. Mugan, Third order differential equations with fixed critical points, Appl. Math. Comput. 208 (2009) 238-248.

[6] M.V. Babich and L.A. Bordag, Projective Differential Geometrical Structure of the Painlevé equations, J. Diff. Eqs. 157 (1999) 452-485.

[7] G. W. Bluman, S. Kumei, Symmetries and Differential Equations, (Springer Verlag, New York, 1989).

[8] F.J. Bureau, Differential equations with fixed critical points, Ann. Mat. Pura Appl. 64 (1964) 229-364.

[9] F. J. Bureau, Differential equations with fixed critical point. II. Ann. Mat. Pura Appl. 66 (1964) 1-116.

[10] F.J. Bureau, Équations différentielles du second ordre en $Y$ et du second degré en $Y$ dont l'intégrale générale est à points critiques fixes., Ann. Mat. Pura Appl. 91 (1972) 163-281.

[11] J. Chazy, Sur les équations différentielles du troisiéme ordre et d'ordre supérieur dont l'intégrale générale a ses points critiques fixes, Acta Math. 34 (1911) 317-385.

[12] J. Chazy, Sur la limitation du degré des coëfficients des équations différentielles algébriques á points critiques fixes, Acta Math. 41 (1916) 29-69.

[13] E.S. Cheb-Terrab, L.G.S. Duarte and L.A.C.P. da Mota, Computer Algebra Solving of Second Order ODEs Using Symmetry Methods, Computer Phys. Comm. 108 (1998) 90-114.

[14] E.S. Cheb-Terrab and A.D. Roche, Integrating Factors for Second Order ODEs, J. Symb. Comp. 27 (1999) 501-519.

[15] F. Contatto and M. Dunajski, Metrisability of Painlevé equations, J. Math. Phys. 59 (2018) 023507.

[16] R. Conte ed., The Painlevé property, one century later, (Springer Verlag, New York, 1999).

[17] R. Conte, A.P. Fordy and A. Pickering, A perturbative Painlevé approach to nonlinear differential equations, Physica D 69 (1993) 33-58.

[18] R. Conte, M. Musette, The Painlevé handbook, (Springer Verlag, New York, 2008).

[19] C.M. Cosgrove, Painlevé classification of all semilinear partial differential equations of the second order. I. Hyperbolic equations of two independent variables, Stud. Appl. Math. 89 (1993) 1-61.

[20] C. M. Cosgrove, Painlevé classification of all semilinear partial differential equations of the second order. II. Parabolic and higher-dimensional equations, Stud. Appl. Math. 89 (1993) 95-151.

[21] C.M. Cosgrove, All binomial-type Painlevé equations of the second order and degree three or higher, Stud. Appl. Math. 90 (1993) 119-187.

[22] C. M. Cosgrove, Painlevé classification problems featuring essential singularities, Stud. Appl. Math. 98 (1997) $355-433$.

[23] C.M. Cosgrove, Chazy classes [IX-XI] of third-order differential equations, Stud. Appl. Math. 104 (2000) 171-228.

[24] C.M. Cosgrove, Higher-order Painlevé equations in the polynomial class. I. Bureau symbol P2, Stud. Appl. Math. 104 (2000) 1-65.

[25] C.M. Cosgrove, Higher-order Painlevé equations in the polynomial class. II. Bureau symbol P1, Stud. Appl. Math. 116 (2006) 321-413.

[26] C.M. Cosgrove, G. Scoufis, Painlevé classification of a class of differential equations of the second order and second degree, Stud. Appl. Math. 88 (1993) 25-87.

[27] H.T. Davies, Introduction to Nonlinear Differential and Integral Equations, (Dover, Mineola, NY, 2010). 
[28] A.M. Escobar-Ruiz, J.C. Lopez-Vieyra and P. Winternitz, Fourth order superintegrable systems separating in Polar Coordinates. I. Exotic Potentials, J. Phys. A: Math. Theor. 50 (2017) 495206.

[29] A.P. Fordy and A. Pickering, Analizing negative resonances in the Painlevé test, Phys. Letts. A 160 (1991) 347-354.

[30] A.P. Fordy and A. Pickering, Generalised Painlevé truncation : expansions in Riccati pseudopotentials, in Symmetries and Integrability of Difference equations, eds. P.A. Clarkson and F.W. Nijhoff, London Mathematical Society LNS 255, pp. 287-298, (CUP, Cambridge, 1999).

[31] B. Gambier, Sur les équations différentielles du second ordre et du premier degré don't l' intégrale génerale est a points critiques fixés, Acta Math. 33 (1910) 1-55.

[32] R. Garnier, Sur des équations différentielles du troisième ordre dont l'intégrale générale est uniforme et sur une classe d'équations nouvelles d'ordre supérieur dont l'intégrale générale a ses points critiques fixes, Annales scientifiques de l'É.N.S. $3^{e}$ série 29 (1912) 1-126.

[33] S. Gravel, Hamiltonians separable in Cartesian coordinates and third-order integrals of motion, J. Math Phys. 45 (2004) 1003-1019.

[34] S. Gravel and P. Winternitz, Superintegrability with third-order integrals in quantum and classical mechanics, $J$. Math Phys. 43 (2002) 5902-5912.

[35] V.I. Gromak, I. Laine, S. Shimomura, Painlevé Differential Equations in the Complex Plane, (De Gruyter, Berlin, 2008).

[36] E.L. Ince, Ordinary Differential Equations, (Dover, Mineola, NY, 1956).

[37] M.D. Kruskal and P. A. Clarkson, The Painlevé-Kowalevski and Poly-Painlevé Tests for Integrability, Stud Appl. Math 86 (1992) 87-165.

[38] M.D. Kruskal, N. Joshi, and R. Halburd, Analytic and asymptotic methods for nonlinear singularity analysis: a review and extensions off tests for the Painlevé property, in Integrability of Nonlinear Systems, Proceedings of CIMPA School, Pondicherry, India, January 8-26, 1996, eds. Y. Kossmann-Schwarzbach, B. Grammaticos and K.M. Tamizhmani, (Springer Verlag, New York 1997).

[39] N.A. Kudryashov, One generalization of the second Painlevé hierarchy, J. Phys. A: Math. Gen. 35 (2002) 93-99.

[40] N.A. Kudryashov, Fourth-order analogies to the Painlevé equations, J. Phys. A: Math. Gen. 35 (2002) 4617-4632.

[41] S. Lie, Klassifikation und Integration von gewohnlichen Differentialgleichunden zwischen x, y die eine Gruppe von Transformationen gestatten. Gessamelte Abhandlungen, Vol. 5, (Teubner, Leipzig, 1924).

[42] I. Marquette, New families of superintegrable systems from k-step rational extensions, polynomial algebras and degeneracies, J. Phys.: Conf. Ser. 597 (2015) 012057.

[43] I. Marquette, M. Sajedi and P. Winternitz, Fourth order superintegrable systems separating in Cartesian coordinates I. Exotic quantum potentials, J. Phys. A: Math. Theor. 50 (2017) 315201.

[44] I. Marquette and P. Winternitz, Superintegrable systems with third-order integrals of motion, J. Phys. A: Math. Theor. 41 (2008) 304031.

[45] M. Mazzocco, Picard and Chazy solutions to Painlevé VI equation, Math. Ann. 321 (2001) 157-195.

[46] U. Mugan, F. Jrad, Painlevé test and higher order differential equations, J. Nonlinear Math. Phys. 9 (2002) 282310.

[47] U. Mugan, F. Jrad, Non-polynomial third order equations which pass the Painlevé test, Z. Naturforsch. A 59 (2004) $163-180$.

[48] M. Noumi, Painlevé Equations through Symmetry, (AMS, Ann Harbour, 2004).

[49] P.J. Olver, Applications of Lie Groups to Differential Equations, (Springer Verlag, New York, 1993).

[50] P. Painlevé, Sur les équations différentielles du second ordre á points critiques fixés, Acta Math. 25 (1902) 1-85.

[51] E. Picard, Mémoire sur la théorie des functions algébriques de deux variables, J. Math. Puré Appl. $4^{\mathrm{e}}$ série, 5 (1889) 135-320.

[52] G. J. Reid, Finding abstract Lie symmetry algebra of differential equations without integrating determining equations, Eur. J. Appl. Math. 2 (1991) 319-340.

[53] G.J. Reid, A.D. Wittkopf and A. Boulton, Reduction of systems of nolinear partial differential equations to simplified involutive form, Eur. J. Appl. Math. 7 (1996) 365-366.

[54] H. Umemura, Galois theory and Painlevé equations, in Théories asymptotiques et équations de Painlevé - Angers, juin 2004, eds. É. Delabaere and M. Loday-Richaud, Séminaires et Congrés 14, pp. 299-339, (SMF, Paris, 2006). 